\title{
Disease-Modifikation und Dauer einer Omalizumab- Therapie bei Patienten mit schwerem allergischen Asthma
}

\section{Disease Modification and Duration of Omalizumab Treatment in Patients with Severe Allergic Asthma}

Autoren

Institute
J. Schreiber ${ }^{1, *}$, M. V. Kopp ${ }^{2, *}$, S. Korn ${ }^{3, *}$, C. Taube ${ }^{4, *}$, R. Buhli, ${ }^{3, *}$

Die Institutsangaben sind am Ende des Beitrags gelistet. eingereicht 14.11 .2013 akzeptiert nach Revision 12.12.2013

\section{Bibliografie}

Dol http://dx.doi.org/ 10.1055/s-0033-1359242

Online-Publikation: 29.1.2014

Pneumologie 2014; 68: 187-192

(c) Georg Thieme Verlag KG

Stuttgart · New York

ISSN 0934-8387

Korrespondenzadresse Prof. Dr. Jens Schreiber Universitätsklinikum der Otto-von-Guericke-Universität Magdeburg

Abteilung für Pneumologie Leipziger Straße 44 39120 Magdeburg jens.schreiber@med.ovgu.de

\section{Zusammenfassung \\ $\nabla$}

Hintergrund und Methodik: Omalizumab ist ein monoklonaler Anti-IgE-Antikörper zur Behandlung des schweren allergischen Asthma bronchiale. Ziel dieser Arbeit war die Bewertung der vorhandenen Evidenz durch ein Expertengremium und die Formulierung daraus resultierender Empfehlungen zu möglichen krankheitsmodifizierenden Effekten und der Dauer der Behandlung mit Omalizumab.

Ergebnisse: Eine direkte oder indirekte Interaktion zwischen Omalizumab und der IgE-Produktion ist wahrscheinlich. Die aufgrund eines pharmakokinetisch-pharmakodynamischen Modells erwarteten IgE-modulierenden Eigenschaften von Omalizumab werden derzeit in der klinischen Anwendung überprüft. Therapieentscheidungen auf Grundlage dieses Modells zu treffen kann allerdings nicht empfohlen werden. Anhand der verfügbaren Evidenz wird ein abschwächender Einfluss von Omalizumab auf Faktoren der strukturellen Veränderungen in den Atemwegen (Airway Remodeling) angenommen. Daten, um eine Empfehlung zur Dosisreduktion oder zum Absetzen von Omalizumab nach Langzeittherapie auszusprechen, fehlen derzeit.

Schlussfolgerungen: Aktuell kann bei Patienten mit schwergradigem allergischen Asthma unter langfristiger Omalizumab-Behandlung aufgrund der vorliegenden klinischen Daten weder eine Dosisreduktion noch ein Absetzen der Therapie empfohlen werden. Für eine klare Beurteilung müssen die Ergebnisse laufender klinischer Studien abgewartet werden.

\section{Abstract \\ $\nabla$}

Background and methods: Omalizumab is a monoclonal anti-IgE-antibody that is used to treat severe allergic asthma. The aim of this review was to evaluate the available evidence in a panel of experts and to provide recommendations on therapy duration with omalizumab.

Results: A direct or indirect interaction between omalizumab and IgE production seems likely. Pharmacokinetic-pharmakodynamic models suggest that omalizumab modulates IgE production. This hypothesis is currently investigated in clinical studies. In addition, available evidence suggests that omalizumab mitigates different factors of airway remodeling. However, based on the currently available data, no recommendations can be given in regard to reduction of dosage or discontinuation of omalizumab in long term treated patients.

Conclusions: Currently, neither dose reductions nor treatment withdrawal can be recommended in patients with severe allergic asthma and long term treatment with omalizumab. Clinical studies addressing these issues are being conducted.

\footnotetext{
* alle Autoren waren maßgeblich beteiligt
} 


\section{Einleitung}

$\nabla$

Omalizumab ist ein rekombinanter, humanisierter, monoklonaler Antikörper, der selektiv menschliches Immunglobulin E (IgE) bindet. Omalizumab ist in mehr als 90 Ländern als additive Therapie zur Verbesserung der Asthma-Kontrolle bei Kindern ab sechs Jahren und Erwachsenen mit schwerem persistierenden allergischen Asthma zugelassen, die einen positiven Hauttest oder eine In-vitro-Reaktivität gegen mindestens ein ganzjährig auftretendes Aeroallergen zeigen und sowohl eine reduzierte Lungenfunktion (bei Erwachsenen; $\mathrm{FEV}_{1}$-forcierte exspiratorische Einsekundenkapazität $<80 \%$ ) haben als auch unter häufigen Symptomen während des Tages oder nächtlichem Erwachen leiden und trotz täglicher Therapie mit hoch dosierten inhalativen Kortikosteroiden und einem lang wirkenden inhalativen Beta ${ }_{2}$ Agonisten mehrfach dokumentierte, schwere Asthma-Exazerbationen haben.

Die Wirksamkeit und Verträglichkeit von Omalizumab wurde in diversen klinischen Studien untersucht, in denen sich ein signifikanter und klinisch relevanter Rückgang der Exazerbationsrate um durchschnittlich 38\% zeigte [1]. Die Rate schwerer Exazerbationen nahm unter Omalizumab sogar um 50\% ab [2,3], und Kontakte mit der Notfallambulanz gingen um $47 \%$ zurück $[1,3]$. In fünf Studien wurde eine umfassende Beurteilung der Behandlungseffektivität durch den behandelnden Arzt durchgeführt (Global Evaluation of Treatment Effectiveness, GETE). Hierbei wurden im Allgemeinen Spirometrie und der maximale exspiratorische Fluss (PEF - peak expiratory flow), Tages- und Nachtsymptome, der Gebrauch der Notfallmedikation und Exazerbationen berücksichtigt. In allen fünf Studien erreichten mit Omalizumab behandelte Patienten im Vergleich zu Plazebo-Patienten entweder eine signifikante Verbesserung oder eine völlige Kontrolle ihrer Asthma-Symptome [4]. Alle Studien belegten eine sehr gute Verträglichkeit der Therapie mit Omalizumab [1 -4]. Omalizumab ist für die Langzeitbehandlung angezeigt. Die geeignete Dosis und Häufigkeit der Anwendung werden aus den vor Behandlungsbeginn gemessenen Gesamt-IgE-Basiswerten (30$1500 \mathrm{IE} / \mathrm{ml}$ ) und dem Körpergewicht (30-150 kg) der Patienten auf Basis einer Dosierungstabelle errechnet [4]. Die Dosierung liegt entsprechend zwischen 75 und $600 \mathrm{mg}$ alle zwei oder vier Wochen. Eine Beurteilung des therapeutischen Ansprechens auf Omalizumab ist bislang lediglich auf der Grundlage klinischer Kriterien im Vergleich zur Situation vor Behandlungsbeginn möglich.

In der klinischen Praxis stellt sich häufig die Frage, ob nach Erreichen der Asthma-Kontrolle und einer längerfristigen Stabilisierung der Erkrankung die Möglichkeit besteht, die Dosis zu reduzieren oder die Therapie mit Omalizumab abzusetzen. Eine Voraussetzung hierfür wäre neben einer anhaltenden Krankheitskontrolle eine über die Beendigung der Therapie hinaus längerfristig anhaltende Modulation der IgE-Produktion durch Omalizumab und das Erreichen eines neuen Equilibriums in der IgEProduktion sowie eine positive Wirkung auf das sogenannte „Airway Remodeling“, d.h. die strukturellen Veränderungen in den Atemwegen der Asthma-Patienten.

Ziel dieses Expertengesprächs war die Bewertung der vorhandenen Evidenz aus klinischen Studien und der Erfahrungen aus der eigenen praktischen Omalizumab-Therapie sowie daraus resultierend die Formulierung von Empfehlungen zur Dauer der Therapie und eventuellen Veränderungen der Dosierung.

\section{Material und Methoden \\ $\nabla$}

Zur Erarbeitung dieser Übersicht und Formulierung von übereinstimmenden Empfehlungen wurde die Methode der „Consensus Development Conference“ in modifizierter Form angewandt [5]. Zur Vorbereitung auf die Konferenz erhielten alle Teilnehmer durch die Novartis Pharma GmbH eine Zusammenfassung der gegenwärtigen Evidenz zum Einfluss von Omalizumab auf den IgEStoffwechsel und das Airway Remodeling. Darüber hinaus wurde den Experten die Liste der während der Konferenz zu diskutierenden Fragen zur Verfügung gestellt.

Während der Konferenz wurden diese Evidenz und diese Fragen gemeinsam mit Vertretern der Novartis Pharma GmbH diskutiert. Daraufhin wurde in einer offenen Diskussion im Expertengremium nach einer Bewertung der vorhandenen Evidenz diese Übersicht erarbeitet und darauf basierend wurden Empfehlungen formuliert.

Um Missverständnisse bei der Protokollierung auszuschließen und um sicherzustellen, dass die Meinung aller beteiligten Experten adäquat berücksichtigt wurde, wurden die Ergebnisse der Konferenz in schriftlicher Form an alle Teilnehmer verteilt und um nochmalige Bewertung gebeten, wodurch eine Zustimmung aller Experten zu der erarbeiteten Übersicht und den getroffenen Empfehlungen erreicht wurde.

\section{Zusammensetzung des Expertengremiums}

Das Expertengremium bestand aus insgesamt fünf anerkannten Pneumologen aus Deutschland und den Niederlanden mit langjähriger Erfahrung in der Behandlung des schweren allergischen Asthma bronchiale. Des Weiteren waren die Experten als Prüfärzte an der klinischen Prüfung von Omalizumab beteiligt und konnten sowohl während dieser Studien als auch in der klinischen Praxis Erfahrung mit der Behandlung mit Omalizumab sammeln.

Die Konsolidierung der protokollierten Kommentare im Anschluss an die Konferenz wurde durch J. Schreiber, Universitätsklinikum der Otto-von-Guericke-Universität, Magdeburg, übernommen.

\section{Ergebnisse}

\section{$\nabla$}

\section{IgE-Modulation durch Omalizumab}

Lowe und Renard modellierten anhand der Daten von rund 2000 mit Omalizumab behandelten Patienten aus sechs randomisierten kontrollierten klinischen Studien (maximale Studiendauer drei Jahre) und einer Beobachtungsstudie über fünf Jahre die Veränderung der IgE-Konzentration und der IgE-Produktionsrate während und nach einer Omalizumab-Langzeittherapie [6]. Das verwendete pharmakokinetisch-pharmakodynamische Feedback-Modell (Lowe-Modell) zeigte, dass Omalizumab die IgEProduktion über Jahre hinweg senkt.

Zu Beginn der Therapie steigt die Serumkonzentration des Gesamt-IgE zunächst an, da die Komplexe aus Omalizumab und IgE aufgrund ihrer längeren Halbwertszeit langsamer abgebaut werden als das freie IgE. Nachdem in den errechneten Modellen nach etwa 5 Jahren ein neues Gleichgewicht (steady state) erreicht wird, wird eine Abnahme der Gesamt-IgE-Konzentration über die Zeit beobachtet. Nach Absetzen der Therapie bleibt die geringere IgE-Produktion zunächst erhalten. Der Abbau der IgE-AntiIgE-Komplexe führt zu einer Abnahme der Gesamt-IgE-Konzentration um das Maß, um das sie zu Anfang der Therapie gestiegen 
ist, sodass sich der Gesamt-IgE-Spiegel nach einer Langzeittherapie mit Omalizumab (hier 3-5 Jahre) unter dem Ausgangsniveau befindet. Sechs Monate bis ein Jahr nach Beendigung der Behandlung steigt die IgE-Produktion wieder an und der Gesamt-IgESpiegel erreicht wieder den Wert vor Beginn der OmalizumabTherapie.

Je länger die Omalizumab-Therapie andauert, desto stärker wird die IgE-Produktion reduziert. Laut Modellierung erreicht daher die Konzentration des Gesamt-IgE den Ausgangswert vor der Therapie umso später, je länger die Therapie andauert [6].

Bislang bestätigte eine Untersuchung an 19 Patienten die auf Grundlage dieses Modells vorhergesagten IgE-modulierenden Eigenschaften von Omalizumab [7]. Die daraus abgeleitete Hypothese wird aktuell in den klinischen Studien EXCELS/XPORT in einer Subgruppe aus 270 Patienten getestet. Die Ergebnisse dieser Analysen werden voraussichtlich 2014 (EXCELS/XPORT) vorliegen.

\section{Moduliert Omalizumab die IgE-Produktion?}

Der vermutete IgE-modulierende Effekt beruht vorwiegend auf einer Modellrechnung. Erste klinische Belege sprechen für diese Hypothese, jedoch reicht die Datenlage momentan noch nicht aus, um diese Frage schlüssig zu beantworten. Insbesondere ist noch ungeklärt, ob eine direkte Beeinflussung der B-Zellen durch Omalizumab stattfindet oder ob ein anderer Wirkmechanismus greift. Unklar sind auch die klinische Relevanz und die mögliche Dauer eines derartigen Effekts.

Eine direkte und/oder indirekte Interaktion zwischen der Verabreichung von Omalizumab und der IgE-Produktion scheint stattzufinden. Ein anti-inflammatorischer Effekt von Omalizumab konnte gezeigt werden: Studien belegen, dass Omalizumab nicht nur das freie IgE reduziert, welches die allergische Kaskade aktiviert $[8,9]$, sondern auch FcERI-Rezeptoren auf Basophilen und dendritischen Zellen herunter reguliert $[10,11]$. Beide Vorgänge führen gemeinsam zu einer Hemmung der IgE-vermittelten Freisetzung proinflammatorischer Mediatoren und damit auch einer erneuten Infiltration mit proinflammatorischen Zellen [12,13]. Wie lange es dauert, bis ein Equilibrium in der IgE-Produktion unter Omalizumab-Therapie erreicht wird, ist derzeit noch unbekannt.

Nach ca. 5 Jahren kann dem Lowe-Modell zufolge ein solches Equilibrium erwartet werden [6]. Klinische Daten liegen jedoch noch nicht vor, sind aber nötig, um diese Hypothesen zu überprüfen. Prinzipiell kann nicht empfohlen werden, Therapieentscheidungen auf Grundlage eines pharmakologischen Modells möglicher Effekte von Omalizumab auf die IgE-Synthese zu treffen. Von der derzeit aktiven XPORT-Studie, welche voraussichtlich 2014 beendet sein wird, wird Aufschluss über die Übertragbarkeit des Modells auf die reale Situation erwartet.

\section{Ist ein Monitoring der IgE-Konzentration im Serum} während einer Therapie mit Omalizumab sinnvoll? Ziel einer Therapie mit Omalizumab ist die Reduktion des freien IgE im Serum unter einen Schwellenwert (ca. $50 \mathrm{ng} / \mathrm{ml}$ ), der wahrscheinlich keine wesentlichen Effekte in der allergischen Reaktionskaskade mehr erwarten lässt. Die initiale Omalizumab-Dosierung orientiert sich an der IgE-Belastung des Patienten, die sich aus dem IgE-Spiegel im Serum und dem Körpergewicht ergibt. Die hierzu in der Fachinformation in Form einer Dosistabelle vorgegebenen Dosierungen erreichen dieses Ziel zuverlässig [4]. Nach Beginn einer Omalizumab-Therapie war bisher ein Monitoring der Serumkonzentration des freien IgE nicht möglich, da kommerziell verfügbare IgE-Messmethoden stets auch IgE als Teil der IgE-Omalizumab-Komplexe erfassen und keinen Rückschluss auf die Konzentration des freien IgE zulassen. Seit Kurzem ist ein Testkit zur Messung des freien IgE im Serum verfügbar [14]. Erste Ergebnisse mit diesem Verfahren legen den Schluss nahe, dass zumindest im therapeutischen Bereich (freies $\operatorname{IgE}<50 \mathrm{ng} / \mathrm{ml}$ ) keine Korrelation zwischen klinischem Therapieerfolg und Serumkonzentration des freien IgE besteht [15]. Vom klinischen Standpunkt aus kann das Monitoring der IgE-Konzentration im Serum (sowohl freies als auch Gesamt-IgE) daher zurzeit weder zur Beurteilung des Therapieansprechens noch zur Steuerung der Therapie (Dosierung) empfohlen werden. Ausschlaggebend für Therapieentscheidungen sind der klinische Zustand des Patienten sowie das Ansprechen auf die Behandlung bezüglich Wirksamkeit und Verträglichkeit. Bei nicht-atopischem Asthma [16] und bei Patienten mit Urtikaria [17] hat sich eine Wirksamkeit von Omalizumab unabhängig vom IgE-Wert des Patienten gezeigt, was jedoch bei allergischem Asthma nicht zwingend auch der Fall sein muss.

Vom wissenschaftlichen Standpunkt aus kann es jedoch durchaus sinnvoll sein, im Rahmen von klinischen Studien ein Monitoring der freien IgE-Konzentration im Serum durchzuführen, beispielsweise um bei Patienten, die nicht auf die Therapie ansprechen (Non-responder), eine Unterdosierung auszuschließen, um die Auswirkungen möglicher Veränderungen des Körpergewichts auf das freie IgE und damit auf die Omalizumab-Dosierung zu erkennen oder um einen modulierenden Einfluss von Omalizumab auf das IgE zu erfassen.

Zum derzeitigen Zeitpunkt liegen jedoch keine aussagekräftigen Daten zur Interaktion zwischen IgE-Konzentration (freies und Gesamt-IgE) und klinischen Symptomen vor. Diese Fragestellungen sollten Bestandteil zukünftiger klinischer Studien sein.

\section{Airway Remodeling}

Asthma ist eine chronische, entzündliche Atemwegserkrankung, die durch eine Überempfindlichkeit und reversible Obstruktion der Atemwege charakterisiert ist. Eine Untergruppe der Patienten entwickelt bei anhaltender unkontrollierter Entzündung eine nur teilweise oder irreversible Verengung der Atemwege mit konsekutivem Verlust an Lungenfunktion [18]. Die strukturellen Veränderungen in den Atemwegen dieser Patienten werden als Airway Remodeling bezeichnet.

Es wird angenommen, dass das Airway Remodeling zum Phänotyp der irreversiblen Bronchialobstruktion und zur Hyperreaktivität der Luftwege beiträgt. Airway Remodeling kann auch mit einem erhöhten Schweregrad der Erkrankung und einem verringerten Ansprechen auf die Therapie einhergehen. Daher wäre eine Verringerung oder gar Rückbildung des Airway Remodelings von entscheidender therapeutischer Bedeutung [19].

Omalizumab führt zu einer Reduktion des freien IgE und reduziert die IgE-Bindungen sowohl an hoch-affine (FceRI) als auch an niedrig-affine (FcERII) IgE-Rezeptoren, was zu einer Abschwächung der allergischen Reaktion führt. Zusätzlich zur Hemmung der Bindung von IgE an Mastzellen, basophile und eosinophile Leukozyten, reguliert Omalizumab die Expression von FceRI auf der Oberfläche der Basophilen und Mastzellen sowie auf antigenpräsentierenden Zellen wie pDC1- und pDC2-Zellen herunter. Dies führt auch zu einer Abnahme der durch die T-Helfer-Zellen vermittelten entzündlichen Immunantwort [10-13,20,21]. Des Weiteren äußert sich der therapeutische Effekt von Omalizumab in einer signifikanten Reduktion der Serumkonzentration von Zytokinen (v.a. IL-13) [22]. 
Patienten, die mit Omalizumab behandelt wurden, zeigten im Vergleich zu Patienten unter konventioneller Therapie eine statistisch signifikante Abnahme der Konzentration von Endothelin-1 (ET-1, dem stärksten bekannten vasokonstriktorischen Stoff) im Atemkondensat (EBC - exhaled breath condensate), eine Reduktion des exhalierten Stickstoffmonoxid (FE(NO)), eine Abnahme des eosinophilen kationischen Proteins (ECP, das im Rahmen von Entzündungsreaktionen von eosinophilen Granulozyten in das umliegende Gewebe abgegeben wird) im Serum und der eosinophilen Leukozyten im Blut. Diese Veränderungen waren begleitet von einer lungenfunktionellen Verbesserung mit einer Zunahme der spirometrischen Parameter (z.B. FEV 1 ). In der Gruppe der mit Omalizumab behandelten Patienten zeigte sich weiterhin eine statistisch signifikante Korrelation zwischen der Abnahme von ET-1 in EBC, der Abnahme von FE(NO), ECP, und der peripheren Eosinophilen-Zahl und der Zunahme der FIV $_{1}$ (inspiratorische Einsekundenkapazität) [23].

In einer Pilot-Studie mit 30 Patienten mit schwerem allergischen Asthma reduzierte Omalizumab nach einer Therapiedauer von 16 Wochen sowohl die bronchiale Wandstärke als auch die entzündlichen Veränderungen der Atemwege [24].

Eine weitere klinische Studie zeigte bei 9 von 11 Patienten eine Verringerung der Dicke der retikulären Basalmembran unter einer Behandlung mit Anti-IgE [25].

Kann auf Grundlage dieser Evidenz geschlussfolgert werden, dass Omalizumab einen Einfluss auf das Airway Remodeling hat?

Die Ergebnisse beider Studien [24,25] berechtigen zu der Hypothese, dass eine Therapie mit Omalizumab einen direkten oder indirekten Effekt auf einige dem Remodeling potentiell zugrunde liegende Pathomechanismen hat.

Die Veränderungen und die ursächlichen Pathomechanismen des Airway Remodelings sind jedoch außerordentlich komplex. Eine sehr gute Evidenz existiert für einen positiven Einfluss von Omalizumab auf die eosinophile Inflammation. Auch die Herabregulation von FceRI auf verschiedenen Zellen und eine Reduktion von proinflammatorischen Zytokinen durch Omalizumab wurde überzeugend nachgewiesen. Außerdem gibt es Hinweise darauf, dass die Wanddicke der Bronchien sowie die Entzündung der Atemwege durch die Therapie mit Omalizumab reduziert wird. Es ist gegenwärtig nicht bekannt, ob Omalizumab diese Effekte über direkte oder indirekte Mechanismen induziert. Die bisher publizierten Daten zum Einfluss von Omalizumab auf Aspekte des Airway Remodelings wurden nur in kleinen Studienpopulationen erhoben und lassen somit kaum aussagekräftige Schlussfolgerungen $\mathrm{zu}$.

Es wurde überzeugend belegt, dass Omalizumab eine positive Wirkung auf den klinischen Verlauf und auf Exazerbationen beim Asthma bronchiale und die asthmatische Entzündung hat [17]. Dass Omalizumab sich günstig auf Parameter des Airway Remodeling auswirkt, wird angenommen, kann aber noch nicht als bewiesen betrachtet werden. Diese Fragestellung ist aktuell Gegenstand einer kontrollierten klinischen Prüfung (eXplore).

\section{Dauer einer Therapie mit Omalizumab}

Die Wirksamkeit von Omalizumab kann auch in der Langzeittherapie als belegt gelten $[26,27]$. Dies wirft die Frage nach der Dauer einer Therapie mit Omalizumab bzw. einer Dosisreduktion in der Langzeittherapie, insbesondere angesichts einer möglichen Modulation der IgE-Produktion (s.o.) auf. Bislang sind die Daten aus klinischen Studien zu diesen Fragen noch sehr spärlich.
In einer französischen Studie wurde die Behandlung mit Omalizumab nach 6 bis 24 Monaten beendet und die Asthma-Kontrolle der Patienten weiter beobachtet. Von insgesamt 110 Patienten war bei 61 (55\%) Patienten eine erneute Behandlung erforderlich, bei einer Behandlungsdauer von unter einem Jahr sogar bei $65 \%$ der Patienten [28].

In einer Studie von Nopp et al. zeigte sich, dass der größte Teil der Patienten (16 von 18; 89\%) mit schwerem allergischen Asthma und einer Katzenhaar- oder Milbenallergie nach 6 Jahren Behandlung mit Omalizumab bis zu 3 Jahre nach Absetzen des Medikaments klinisch stabil blieb [29,30]. Eine Voraussetzung für die Beendigung der Omalizumab-Therapie war dabei eine über lange Zeit stabile Asthma-Kontrolle der Patienten. Kritisch anzumerken ist, dass aus der Publikation nicht hervorgeht, in wieweit von den Patienten eine Allergen-Karenz realisiert wurde.

\section{Kann eine Dosisreduktion von Omalizumab empfohlen werden/sinnvoll sein?}

Eine auf klinischer Evidenz basierende Antwort auf diese Frage ist derzeit nicht möglich.

Außer in Fällen von drastischem Gewichtsverlust, welcher eine Dosisreduktion unumgänglich macht (siehe Dosierungs-Tabelle der Fachinformation [4]), kann eine Reduzierung der Omalizumab-Dosis unter die in der Dosierungs-Tabelle angegebene Dosis nicht empfohlen werden. Insbesondere nach kürzerer Therapiedauer muss davon ausgegangen werden, dass es in der Folge zu einer Verschlechterung der Asthma-Kontrolle kommt [31]. Die Auswirkungen der Dosisreduktion nach einer langjährigen Therapie insbesondere auf die Entzündungsprozesse und einer eventuellen Progression des Airway Remodelings sind noch unbekannt. Die Frage, ob die Steuerung der Therapie (Omalizumab-Dosierung) durch Monitoring der Konzentration des freien IgE im Serum im Verlauf die Therapieergebnisse verbessert oder eine Dosisreduktion erlaubt (z.B. Abnahme der IgE-Produktion) bzw. phasenweise Erhöhungen der Dosis begründet (z.B. zusätzliche saisonale Allergen-Exposition), sollte in einer kontrollierten klinischen Prüfung untersucht werden.

Wie lange sollte mit Omalizumab behandelt werden? Kann eine Beendigung der Behandlung mit Omalizumab empfehlenswert sein?

Eine auf klinische Evidenz basierte Antwort auf diese Frage ist derzeit nicht möglich.

Anhand der bisher gewonnenen Daten $[26,30]$ ist das Absetzen einer wirksamen Therapie, außer bei schweren Unverträglichkeitsreaktionen, generell nicht empfehlenswert. Es ist davon auszugehen, dass bei der Mehrzahl der auf eine Therapie mit Omalizumab klinisch ansprechenden Patienten eine Beendigung der Therapie nach 1-2 Jahren zu einer Verschlechterung der Asthma-Kontrolle führt $[28,31]$. Allerdings besteht die Möglichkeit, dass nach einer deutlich längeren Therapiedauer (nach ca. 6 Jahren) positive Effekte auch bei Absetzen der anti-IgE-Therapie erhalten bleiben $[29,30]$. Die Therapie sollte darauf ausgerichtet werden, eine möglichst gute Krankheitskontrolle zu erreichen und die Kortikosteroidtherapie auf ein Minimum bei Beibehaltung der Krankheitskontrolle zu reduzieren. Während bei erwachsenen Patienten vor allem die Reduktion und möglichst ein Absetzen der systemischen Steroide angestrebt wird, ist es bei pädiatrischen Patienten ebenfalls besonders wichtig, die inhalative Steroiddosis zu reduzieren. Der Gebrauch inhalativer Steroide im prä-pubertären Alter resultiert nachweisbar in Wachstumsstörungen [31]. 
Individuell, insbesondere wenn triftige Gründe (z.B. Nebenwirkungen) für eine Beendigung der Therapie sprechen, kann eine Unterbrechung der Therapie mit Verlaufsbeurteilung unter engmaschiger Überwachung der Asthma-Kontrolle sinnvoll sein. Die klinische Erfahrung im Omalizumab-Studienprogramm hat gezeigt, dass es nach Beendigung der Therapie meist zu einer Verschlechterung der Asthma-Kontrolle und -Symptomatik kommt, die der Situation vor Beginn der Therapie entspricht [32]. Die Entscheidung sollte immer nach sorgfältiger Abwägung der individuellen Vor- und Nachteile, nach sorgfältiger klinischer und lungenfunktioneller Untersuchung und unter Berücksichtigung ggf. weiterer individueller Faktoren (wie z.B. Allergen-Exposition, Umfeld des Patienten) im gegenseitigen Einvernehmen zwischen behandelndem Arzt und Patient erfolgen (vgl. GINA globale Richtlinie [33]). Eine erneute Initiierung der Omalizumab-Therapie ist in solchen Situationen jederzeit und nach aktuellem Kenntnisstand ohne Einbußen an Wirksamkeit möglich. Die Konsequenzen eines Abbruchs der Therapie mit Omalizumab sind Gegenstand derzeit aktiver klinischer Studien (z.B. XPORT).

\section{Zusammenfassung}

Es wird angenommen, dass Omalizumab einen Effekt auf die IgE-Modulation und das Airway Remodeling hat, allerdings fehlen zum derzeitigen Zeitpunkt aussagekräftige Daten, um diese Annahme zu belegen. Empfehlungen zur Dosisreduktion oder zum Absetzen von Omalizumab können nicht gegeben werden. Alle Fragestellungen werden im Rahmen von laufenden und geplanten klinischen Studien behandelt.

\section{Danksagung}

Die Autoren danken Frau Alexandra Rachfahl und Frau Franziska Meister (beide Novartis Pharma GmbH, Nürnberg) für die editorische Unterstützung beim Erstellen des Manuskripts.

Außerdem danken die Autoren Herrn Felix Krippner, Frau Dr. Kristin Lerche, Frau Dr. Sandra Schreiber (Novartis Pharma $\mathrm{GmbH}$, Nürnberg) sowie Frau Dr. Tamara Kiechle, Herrn Robert Maykut und Herrn Phil Lowe (Novartis AG, Basel) für die Darstellung der aktuellen Daten und Informationen zu Omalizumab.

\section{Interessenkonflikt}

$\nabla$

Die an dieser Übersicht beteiligten Autoren haben in den letzten Jahren mehrfach von Fa. Novartis Beratungshonorare, Honorare für Vorträge und Unterstützungen bei Kongressbesuchen im Inund Ausland erhalten.

\footnotetext{
Institute

${ }^{1}$ Universitätsklinikum der Otto-von-Guericke-Universität Magdeburg, Abteilung für Pneumologie

2 Universitätsklinikum Schleswig-Holstein, Campus Lübeck, Klinik für Kinder- und Jugendmedizin, Pädiatrische Pneumologie und Allergologie, Airway Research Center North (ARCN), Mitglied des Deutschen Zentrums für Lungenforschung (DZL)

${ }^{3}$ Universitätsmedizin der Johannes-Gutenberg-Universität, Mainz, III. Medizinische Klinik und Polyklinik, Schwerpunkt Pneumologie ${ }^{4}$ Leiden University Medical Center, Dept. of Pulmonology
}

\section{Literatur}

1 Bousquet J, Cabrera P, Berkman $N$ et al. The effect of treatment with omalizumab, an anti-IgE antibody, on asthma exacerbations and emergency medical visits in patients with severe persistent asthma. Allergy 2005; 60: $302-308$

2 Humbert M, Beasley $R$, Ayres J et al. Benefits of omalizumab as add-on therapy in patients with severe persistent asthma who are inadequately controlled despite best available therapy (GINA 2002 step 4 treatment): INNOVATE. Allergy 2005; 60: 309-316

3 Holgate S, Buhl R, Bousquet J et al. The use of omalizumab in the treatment of severe allergic asthma: A clinical experience update. Respir Med 2009103: 1098-1113

4 Novartis Pharma. Fachinformation Xolair. 2012; Ref Type: Generic

5 Murphy MK, Black NA, Lamping DL et al. Consensus development methods, and their use in clinical guideline development. Health Technol Assess 1998; 2: i-88

6 Lowe PJ, Renard D. Omalizumab decreases IgE production in patients with allergic (IgE-mediated) asthma; PKPD analysis of a biomarker, total IgE. Br J Clin Pharmacol 2011; 72: $306-320$

7 Hanf G, Brachmann I, Kleine-Tebbe J et al. Omalizumab decreased IgErelease and induced changes in cellular immunity in patients with allergic asthma. Allergy 2006; 61: 1141 - 1144

8 van Rensen EL, Evertse CE, van Schadewijk WA et al. Eosinophils in bronchial mucosa of asthmatics after allergen challenge: effect of anti-IgE treatment. Allergy 2009; 64: 72-80

9 Shields $R L$, Whether WR, Zioncheck $K$ et al. Inhibition of allergic reactions with antibodies to IgE. Int Arch Allergy Immunol 1995; 107: $308-312$

10 Prussin C, Griffith DT, Boesel KM et al. Omalizumab treatment downregulates dendritic cell FcepsilonRI expression. J Allergy Clin Immunol 2003; 112: $1147-1154$

11 MacGlashan DWJr, Bochner BS, Adelman DC et al. Down-regulation of $\mathrm{Fc}$ (epsilon)RI expression on human basophils during in vivo treatment of atopic patients with anti-IgE antibody. J Immunol 1997; 158: 1438 1445

12 Massanari M, Holgate ST, Busse WW et al. Effect of omalizumab on peripheral blood eosinophilia in allergic asthma. Respir Med 2010; 104: $188-196$

13 Oliver JM, Tarleton CA, Gilmartin L et al. Reduced FcepsilonRI-mediated release of asthma-promoting cytokines and chemokines from human basophils during omalizumab therapy. Int Arch Allergy Immunol 2010; $151: 275-284$

14 Strohner P, Korn S, Buhl R et al. The recovery-ELISA - a novel assay technique to monitor therapy with humanized antibodies: the example of omalizumab. J Immunoassay Immunochem 2013; 34: 83 - 93

15 Korn S, Haasler I, Fliedner F et al. Monitoring free serum IgE in severe asthma patients treated with omalizumab. Respir Med 2012; 106: $1494-1500$

16 Garcia G, Magnan A, Chiron $R$ et al. A proof-of-concept randomizedcontrolled trial of omalizumab in patients with severe difficult to control nonatopic asthma. Chest 2013. In press

17 Maurer M, Rosen K, Hsieh $\mathrm{HJ}$ et al. Omalizumab for the treatment of chronic idiopathic or spontaneous urticaria. N Engl J Med 2013; 368: 924-35

18 Shifren A, Witt C, Christie C et al. Mechanisms of remodeling in asthmatic airways. J Allergy (Cairo) 2012; 2012: 316049

19 Rabe KF, Calhoun WJ, Smith $N$ et al. Can anti-IgE therapy prevent airway remodeling in allergic asthma? Allergy 2011; 66: 1142-1151

20 Noga 0, Hanf G, Brachmann I et al. Effect of omalizumab treatment on peripheral eosinophil and T-lymphocyte function in patients with allergic asthma. J Allergy Clin Immunol 2006; 117: 1493 - 1499

21 Galli SJ, Tsai M. IgE and mast cells in allergic disease. Nat Med 2012; 18 : $693-704$

22 Noga 0 , Hanf G, Kunkel G. Immunological and clinical changes in allergic asthmatics following treatment with omalizumab. Int Arch Allergy Immunol 2003; 131: 46-52

23 Zietkowski Z, Skiepko R, Tomasiak-Lozowska MM et al. Anti-IgE therapy with omalizumab decreases endothelin-1 in exhaled breath condensate of patients with severe persistent allergic asthma. Respiration 2010; 80: $534-542$

24 Hoshino M, Ohtawa J. Effects of adding omalizumab, an anti-immunoglobulin E antibody, on airway wall thickening in asthma. Respiration 2012; 83: $520-528$ 
25 Riccio AM, Dal Negro RW, Micheletto $C$ et al. Omalizumab modulates bronchial reticular basement membrane thickness and eosinophil infiltration in severe persistent allergic asthma patients. Int J Immunopathol Pharmacol 2012; 25: $475-84$

26 Pace E, Ferraro M, Bruno A et al. Clinical benefits of 7 years of treatment with omalizumab in severe uncontrolled asthmatics. J Asthma 2011; 48: $387-392$

27 Eisner MD, Zazzali JL, Miller MK et al. Longitudinal changes in asthma control with omalizumab: 2-year interim data from the EXCELS Study. J Asthma 2012; 49: $642-648$

28 Molimard M, LeGros V, Bourdeix I. Observational study in severe asthmatic patients after discontinuation of omalizumab for good asthma control. Eur Respir J 2012; 40 (Suppl. 56): 856
29 Nopp A, Johansson SG, Ankerst J et al. CD-sens and clinical changes during withdrawal of Xolair after 6 years of treatment. Allergy 2007; 62: $1175-1181$

30 Nopp A, Johansson SG, Adedoyin J et al. After 6 years with Xolair; a 3-year withdrawal follow-up. Allergy 2010; 65: 56-60

31 Kelly HW, Sternberg AL, Lescher R et al. Effect of Inhaled Glucocorticoids in Childhood on Adult Height. N Engl J Med 2012; 367: 904 - 912

32 Slavin RG, Ferioli C, Tannenbaum SJ et al. Asthma symptom re-emergence after omalizumab withdrawal correlates well with increasing IgE and decreasing pharmacokinetic concentrations. J Allergy Clin Immunol 2009; 123: 107 - 113

33 Global Initiative for Asthma. Global Strategy for Asthma Management and Prevention 2011. www ginasthma.org [cited 2012 Sep 20] 